\title{
OPERATION AND PERFORMANCE OF THE SPring-8 STORAGE RING
}

\author{
S. Date, K. Kumagai, N. Kumagai, M. Masaki, T. Nakamura, H. Ohkuma*, \\ T. Ohshima, K. Soutome, S. Takano, M. Takao, and H. Tanaka, JASRI/SPring-8, \\ 323-2, Mihara, Mikazuki-cho, Sayo-gun, Hyogo 679-5198, Japan \\ K. Tamura, The Institute of Physical and Chemical Research (RIKEN), \\ 2-1, Hirosawa, Wako, Saitama 351-0106, Japan
}

\begin{abstract}
Two-year operation experiences of the SPring-8 storage ring are presented. The commissioning of the storage ring was successfully completed, with the achievement of performance far beyond the target specifications. The beam lifetime is about 60 hours at $100 \mathrm{~mA}$ in the multi bunch mode. The $7 \mathrm{~nm}$.rad of the emittance measured for the horizontal plane is close to the theoretical value. From the beginning of the operation, vertical emittance is extremely small, about $10 \mathrm{pm} \cdot \mathrm{rad}$ and the coupling is about $0.2 \%$. Furthermore, the procedures for decreasing the coupling have been tested. Purity in the few $10^{-6}$ range is routinely achieved in the single and several bunch operation of user service mode. We achieved a good orbit stability at source points, about 5 and 1 micron in an r.m.s. value respectively for horizontal and vertical planes. The beam performance of the SPring-8 storage ring is presented.
\end{abstract}

\section{INTRODUCTION}

Beam commissioning of the storage ring was started since March 13th and the first stage of it was successfully completed by accumulating the electron beams of about $20 \mathrm{~mA}$ one month later. This quick tuning was mainly due to the high completion of hardware. Especially, the precision of magnet alignment was so high that no steering magnet was used to store the first beam in the ring [1]. The major milestones up to now are listed Table 1 .

Table 1 : Progress of the SPring-8 Storage Ring

\begin{tabular}{cl}
\hline Date & Milestones \\
\hline 1997 & \\
$3 / 13$ & Start of SSBT tuning \\
$3 / 13$ & Start of storage ring beam commissioning \\
$3 / 14$ & Completion of first turn \\
$3 / 25$ & Capture of electron beams in RF buckets \\
$3 / 26$ & First observation of photon beams \\
& $\quad$ from a bending magnet \\
$4 / 17$ & Stored current of 19.6 mA \\
& (lifetime 3 hrs with ID gap fully opened) \\
$5 / 14$ & Start of beamline commissioning (BL02B1 and \\
& BL47IN) \\
$5 / 16$ & Operation with ID minimum gap of 8 mm \\
& \\
\hline & Corresponding Author; Email:ohkuma@ spring8.or.jp
\end{tabular}

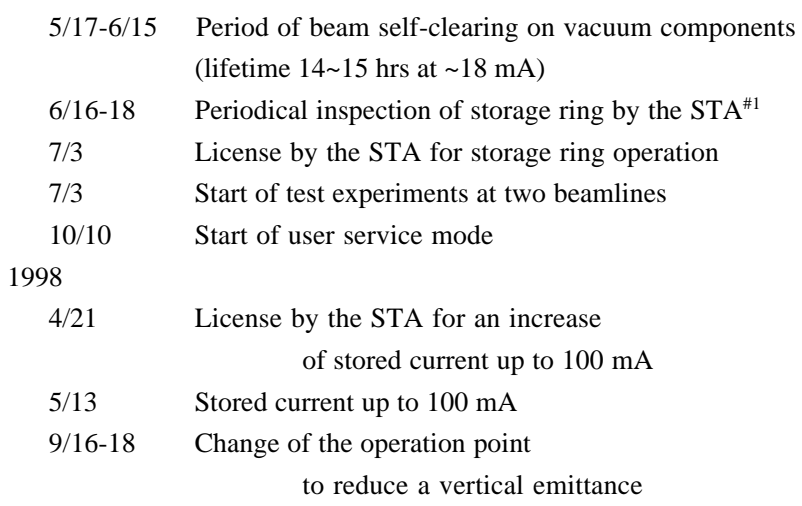

\#1 the Science and Technology Agency

\section{OPERATION}

\subsection{Injection efficiency}

We tried to find the best parameter set of septum and bump magnet strengths to make injection efficiency as high as possible. The auxiliary DC bump was made in the injection part if necessary to reduce a coherent amplitude. At present, the efficiency of $80-90 \%$ has been achieved.

\subsection{Operation time}

The storage ring is operated on three weeks basis (1 cycle), with $38 \times 8$ hour shifts for user service mode, another $6 \times 8$ hour shifts for machine studies, and about 48 hours for machine tuning and for beamline tuning.

In 1998, a total operation time of the storage ring is 4190 hours, and 2624 hours $(62.6 \%)$ has been delivered to the users during the year 1998. The down time is 110 hours, $2.6 \%$ of the total operation time. The most significant incident in 1998 was an user time interruption of 15 hours when the breakdown of the power supply of the sextupole magnets happened. During the last stage of 1998 , user time was often disturbed when an action of the interlock system of the magnet and photon-absorber occurred due to a drop of flow rate of the cooling water.

Typical operation is shown in Fig. 1, when the beam delivered 232 hours without a beam loss.

\subsection{Filling modes}

A $62.3 \%$ of the total user time were delivered in the multi-bunch mode as shown in Fig. 2. In the first half of 


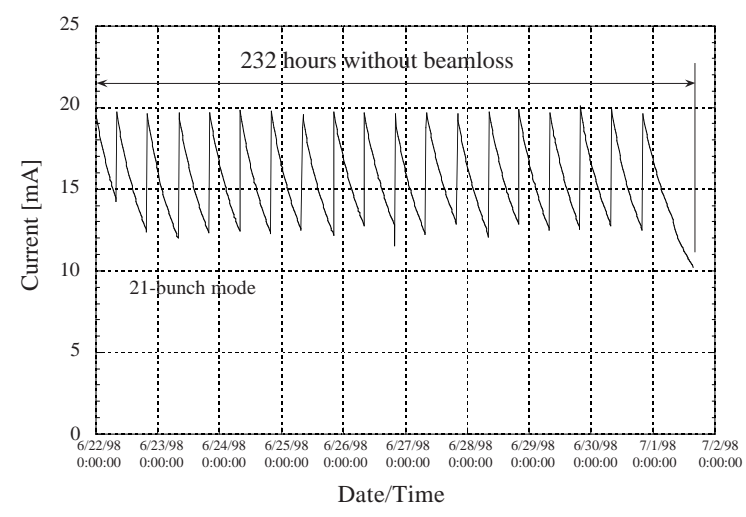

1998, a full filling mode was used for the user time operation. After the summer shutdown period of 1998, a 2/3-filling mode began to be used for the user time operation, where two third of the 2436 available RF buckets are filled continuously with electron.In the SPring-8 storage ring, the beam lifetime in the uniform filling mode is significantly longer than that in the partial filling mode. This origin may be that the electron beam size is growth by the instability due to an ion-trapping effect.

Figure 1: Beam delivery 232 hours without beamloss.

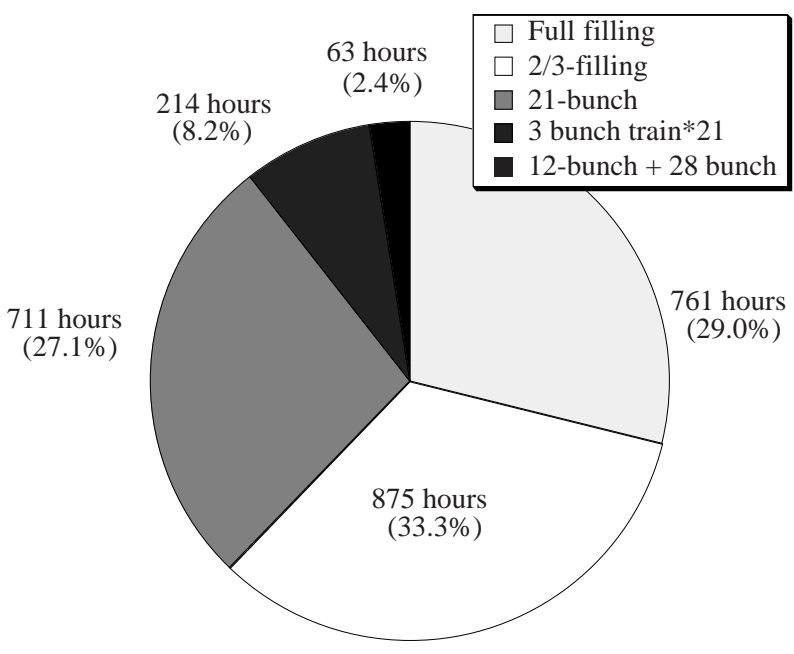

Figure 2: Filling modes for user time during 1998.

Nevertheless, the several bunch modes is increasing. For example, there were many time (724 hours) in 21bunch mode (21 equally spaced bunches) between May and early in July 1998. Another several bunch modes are the 21-bunch train mode (21 equally spaced 3- or 7-bunches trains), 10-bunch + partially filled multi-bunch, and so on. Since we can select an arbitrary RF bucket among 2436 ones at each injection, the filling pattern in the storage ring can be controlled easily [2]. 1 or 0.5
$\mathrm{mA} / \mathrm{bunch}$ are stored, and purities in the low $10^{-6}$ range are routinely achieved in the user time operation. The maximum current per bunch is about $12 \mathrm{~mA}$ in machine studies.

\section{PERFORMANCE OF THE STORAGE RING}

The over-all performance are listed in Table 2.

Table 2 : Performance of the SPring-8 Storage Ring

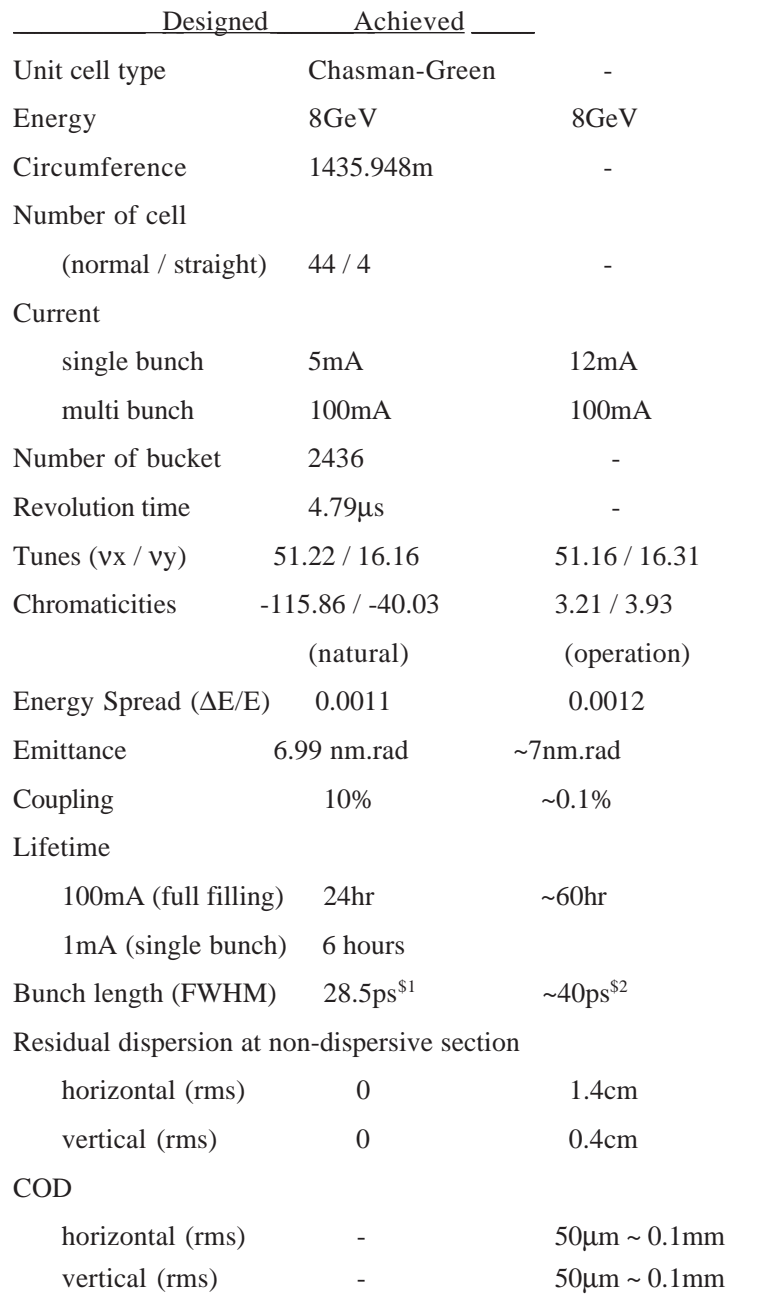

$\$ 1$ The design value is estimated at the $\mathrm{Vrf}=17 \mathrm{MV}$.

$\$ 2$ The achieved value is measured at $\mathrm{Vrf}=11.6 \mathrm{MV}$.

\subsection{Optics}

The lattice structure of the SPring- 8 storage ring is of double-bend achromat type, and there are 48 straight sections where the dispersion vanishes.

At the normal operation of the storage ring, the "hybrid" optics was used. In the "hybrid" optics, the horizontal betatron function $\beta_{\mathrm{X}}$ takes a large value (about $20 \mathrm{~m}$ ) and a small value (about $1 \mathrm{~m}$ ) alternately in the straight sections.

The storage ring is routinely operated near the 
coupling resonance $\left(v_{x}=51.25, v_{y}=16.32\right)$ with a coupling ratio of about $0.4 \%$ before September 1998. After the summer shutdown period of 1998, the procedures for decreasing the coupling, i.e. tuning the mode tune separation to change the operation point, have been successfully performed. New operation point is $\left(v_{x}=51.15\right.$, $\left.v_{y}=16.31\right)$. At this operation point, the coupling ratio is estimated to be around $0.06 \%$. When compared with the $0.4 \%$, this reduction of the coupling ration gives an equivalent gain of a factor of 0.4 in vertical beam size.

After the winter shutdown of 1999 , in order to cure a short beam lifetime due to the Touschek effect in the several bunch mode, at the user time the storage ring have been operated with the enlarged coupling ratio of $0.2 \%$, which resulted in $\sim 1.5$ times increase of the beam lifetime.

The "high-beta like" optics in which $\beta_{\mathrm{x}}$ and $\beta_{\mathrm{y}}$ take a same value $\left(\beta_{\mathrm{X}}: 25 \mathrm{~m}\right.$, and $\left.\beta_{\mathrm{y}}: 3.7 \mathrm{~m}\right)$ in all the straight sections have been tested in machine studies. The "highbeta like" optics has a high symmetry and optimization for insertion device in all the straight section, compared with the "hybrid" optics. Betatoron tunes are $v_{x}=43.16$ and $v_{y}=21.31$. Two kinds of optics are shown in Fig. 3.

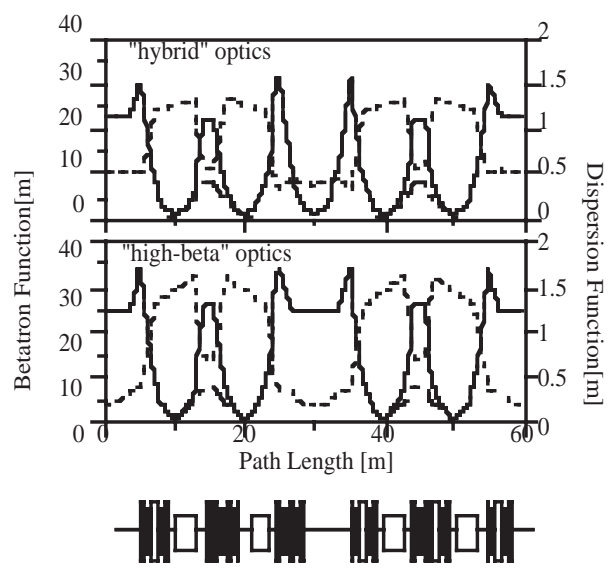

Figure 3: Optics of the SPring-8 storage ring.

\subsection{Beam Reproducibility and Stability}

The fill-to-fill and cycle-to-cycle reproducibility of the storage ring parameters are assured by strictly following a prescribed start-up sequence that includes conditioning of all magnets at start-up or after a failure that shuts down a power supply. The power supplies of magnets are continuously turned on between cycles, 4 or 5 days. The reproducibility is checked by measuring the betatron tunes, COD, and dispersion which are sensitive to both magnet setting. In order to stabilize the orbit of the storage ring, a periodic and global orbit correction is now routinely used in user time. Figure 4 shows the amplitude changes of betatron tune-harmonics (51th for horizontal and 16th for vertical) of the COD with and without the correction of the global orbit distortion [3].

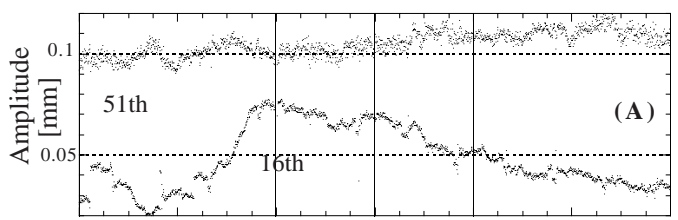

$\begin{array}{lllllll}\text { Jun/20/98 } & \text { Jun/21/98 } & \text { Jun/22/98 } & \text { Jun/23/98 } & \text { Jun/24/98 } & \text { Jun/25/98 } & \text { Jun/26/98 }\end{array}$

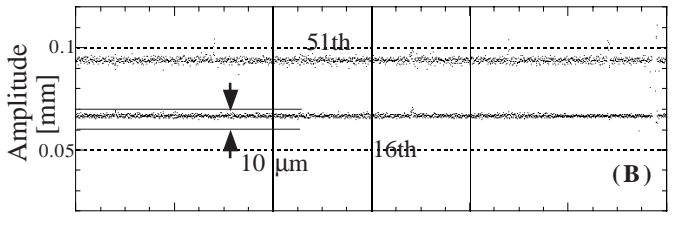

Dec/4/98 Dec/5/98 Dec/6/98 $\quad$ Dec/7/98 $\quad$ Dec/8/98 $\quad$ Dec/9/98 $\quad$ Dec/10/98

Figure 4: Amplitude changes of tune-harmonics (51th for horizontal and 16th for vertical) of the COD for 6 days. The upper (A) and lower (B) graphs show respectively the changes without and with the periodic correction of global orbit correction

\subsection{VACUUM CONDITIONING AND BEAM LIFETIME [4]}

The averaged pressure readings of the storage ring are $\leq 1 \times 10^{-8} \mathrm{~Pa}$ without electron beam, the order of $10^{-7} \mathrm{~Pa}$ with a beam current of $70 \mathrm{~mA}$. Since then an integrated beam dose of 190Ah has been achieved up to now, and correspondingly, the dynamic pressure rise per stored beam current $(\Delta P / I)$ decreased by two orders of magnitude. An integrated beam dose of 190Ah and a beam lifetime of 60 hours at $100 \mathrm{~mA}$ of the beam current were achieved in the multi-bunch mode after two years' operation. The lifetime in the several bunches mode is limited by Touschek effect. Touschek lifetime is about 6 hours at the single bunch operation (1mA/bunch) of the nominal machine parameter of the SPring- 8 storage ring [5]. As the total lifetime $(\tau)$ in the 2/3-filling mode is 65 hours at the beam current of $70 \mathrm{~mA}$, gas scattering lifetime is estimated $\tau_{\mathrm{g}} \approx 140$ hours.

\section{REFERENCES}

[1] H. Tanaka, N. Kumagai, and K. Tsumaki, Nucl. Instr. and Meth., A313 (1992) 529.

[2] T. Ohshima, et al., Proc. of the 11th Symp. on Accelerator Sci. and Tech., Harima, Japan, 1997, 359.

[3] H. Tanaka, et al., "Orbit Stabilization in SPring-8 Storage Ring", presented in this proceedings.

[4] H. Ohkuma, et al., "Vacuum Performance and Beam Lifetime of the SPring- 8 Storage Ring", presented in this proceedings.

[5] M. Takao, et al., "Estimation of Betatoron Coupling and Vertical Dispersion for Spring-8 Storage Ring", presented in this proceedings. 